Published in final edited form as:

Clin Cancer Res. 2021 April 01; 27(7): 2038-2049. doi:10.1158/1078-0432.CCR-20-3262.

\title{
Preclinical modeling of surgery and steroid therapy for glioblastoma reveals changes in immunophenotype that are associated with tumor growth and outcome
}

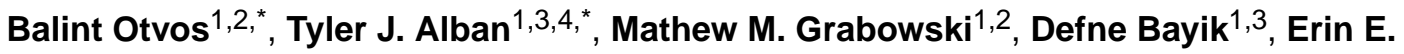 \\ Mulkearns-Hubert ${ }^{1}$, Tomas Radivoyevitch ${ }^{5}$, Anja Rabljenovic ${ }^{1}$, Sarah Johnson ${ }^{1}$, Charlie \\ Androjna $^{6}$, Alireza M. Mohammadi ${ }^{2,7,10}$, Gene H. Barnett ${ }^{2,7,10}$, Manmeet S. Ahluwalia ${ }^{2,7,10}$, \\ Michael A. Vogelbaum ${ }^{8}$, Peter E. Fecci ${ }^{9}$, Justin D. Lathia ${ }^{1,3,4,7,10}$ \\ ${ }^{1}$ Department of Cardiovascular and Metabolic Sciences, Lerner Research Institute, Cleveland \\ Clinic, Cleveland, Ohio, 44195, USA \\ ${ }^{2}$ Department of Neurosurgery, Cleveland Clinic, Cleveland, Ohio, 44195, USA \\ ${ }^{3}$ Case Comprehensive Cancer Center, Case Western Reserve University, Cleveland, Ohio, \\ 44106, USA \\ ${ }^{4}$ Department of Molecular Medicine, Cleveland Clinic Lerner College of Medicine of Case \\ Western Reserve University, Cleveland, Ohio, 44195, USA \\ ${ }^{5}$ Department of Quantitative Health Sciences, Lerner Research Institute, Cleveland Clinic, \\ Cleveland, Ohio, 44195, USA \\ ${ }^{6}$ Department of Biomedical Engineering, Lerner Research Institute, Cleveland Clinic, Cleveland, \\ Ohio, 44195, USA \\ ${ }^{7}$ Rose Ella Burkhardt Brain Tumor and Neuro-Oncology Center, Cleveland Clinic, Cleveland, Ohio, \\ 44195, USA \\ ${ }^{8}$ Department of NeuroOncology, Moffitt Cancer Center, Tampa, Florida, 33612, USA \\ ${ }^{9}$ Department of Neurosurgery, Duke University Hospital, Durham, NC, USA \\ ${ }^{10}$ Cleveland Clinic Lerner College of Medicine of Case Western Reserve University, Cleveland, \\ Ohio, 44195, USA
}

\section{Abstract \\ Purpose: Glioblastoma (GBM) immunotherapy clinical trials are generally initiated after standard-of-care treatment, including surgical resection, perioperative high-dose steroid therapy,}

\footnotetext{
Corresponding author: Justin D. Lathia, Ph.D., Lerner Research Institute, 9500 Euclid Ave., NC10, Cleveland, OH 44195, Phone: 216-445-7475, lathiaj@ccf.org.

* Denotes equal contribution

Author Contributions

BO, TA, MG, MV, PF, and JL provided conceptualization and design. BO, TA, DB, AR, SJ, CA, performed the experiments. BO, TA, MG, DB, AR CA, TR, AM, MA, MV, PF, JL analyzed the data. BO, TA, MG, EM, TR, MV, PF, JL wrote the manuscript. BO, JL provided financial support. All authors provided final approval of the manuscript.

Conflicts of Interest: The authors declare no potential conflicts of interest.
} 
chemotherapy, and radiation treatment, has either begun or failed. However, the impact of these interventions on the anti-tumoral immune response is not well studied. While discoveries regarding the impact of chemotherapy and radiation on immune response have been made and translated into clinical trial design, the impact of surgical resection and steroids on the anti-tumor immune response has yet to be determined.

Experimental Design: We developed a murine model integrating tumor resection and steroid treatment and used flow cytometry to analyze systemic and local immune changes. These mouse model findings were validated in a cohort of 95 primary GBM patients.

Results: Using our murine resection model, we observed a systemic reduction in lymphocytes corresponding to increased tumor volume and decreased circulating lymphocytes that was masked by dexamethasone treatment. The reduction in circulating T cells was due to reduced CCR7 expression, resulting in T-cell sequestration in lymphoid organs and the bone marrow. We confirmed these findings in a cohort of primary GBM patients and found that prior to steroid treatment, circulating lymphocytes inversely correlated with tumor volume. Lastly, we demonstrated that peripheral lymphocyte content varies with progression-free and overall survival, independent of tumor volume, steroid use, or molecular profiles.

Conclusions: These data reveal that prior to intervention, increased tumor volume corresponds with reduced systemic immune function and that peripheral lymphocyte counts are prognostic when steroid treatment is taken into account.

\section{Introduction}

Glioblastoma (GBM), a WHO grade IV glioma, is treated with standard-of-care therapies including maximal safe surgical resection, steroids, and concomitant radiation and chemotherapy with temozolomide. Despite the short-term efficacy of these approaches, the 5 -year survival rate remains only 5\%(1). Following the recent success of immunotherapy in cancers such as high-grade melanoma and lung cancer, there is currently an extensive clinical trial effort to produce similar anti-tumor immune responses in patients with $\operatorname{GBM}(2)$. Thus far, these trials have largely failed to impact survival in GBM patients, but they have successfully drawn attention to the potent local and systemic immune suppression elicited by GBM(2-6). In seeking to develop better immune therapies for GBM, many have logically sought to understand how standard-of-care chemotherapy(7-10) and radiation(1113) impact the immune system and the tumor immune microenvironment. Although neoadjuvant immunotherapy prior to surgical resection may lead to more effective treatment responses(14), the impact of standard surgical resection and steroid treatment otherwise remains largely unexplored. Recently, two studies highlighted the importance of investigating steroid use in GBM: these studies found that the dose of dexamethasone reduces immunotherapy response in mouse models, and a clinical trial showed a similar reduction in overall survival due to steroid treatment $(15,16)$. As part of these studies, it was also observed that low-dose anti-VEGF treatment reduced edema, providing an alternative to dexamethasone that can be rapidly applied to future clinical trials $(15,16)$. However, these studies do not take into account the impact of surgical resection of the bulk tumor or the size of the tumor on immune response. The singular effects of steroids versus surgical resection have not been specifically addressed in prior studies, as every patient who undergoes 
surgical resection also receives steroid treatment to reduce edema. In addition, patients typically undergo chemotherapy and radiation shortly after surgery, further complicating our understanding of how surgery and steroids impact the anti-tumor immune response.

To investigate the immunological changes that occur due to standard-of-care treatment, we developed a mouse model of surgical resection that also employs a clinically relevant steroid dose, similar to those shown in the same mouse models to reduce immunotherapy activity(15). We utilized two syngeneic murine GBM models (GL261 and CT-2A(17)) and found that surgical resection alone elicited a subsequent reduction in peripheral $\mathrm{T}$ cells, as did treatment with steroids. Additionally, we identified a negative correlation between the number of $\mathrm{T}$ cells in the circulation and tumor volume. We confirmed similar correlations in a GBM patient cohort, where patients who did not receive steroid treatment exhibited a negative correlation between peripheral blood lymphocyte counts and tumor volume. Initial lymphocyte count was a significant predictor of progression-free- and overall survival in multivariate models. Taken together, these studies demonstrate that the immune status of GBM patients is impacted by surgical resection and tumor size, two major factors impacting all GBM patients that have not been previously described and should be considered in the design of immunotherapy clinical trials.

\section{Materials and Methods}

\section{Syngenic Tumor Resection Models}

GL261 mice were acquired from the NCI and CT-2A mice were obtained from Dr. Thomas Seyfried (Boston University). Six-week-old aged-matched male C57BL/6J mice from Jackson Labs (000664) were anesthetized using isoflurane and then intracranially injected into the left cerebral hemisphere with 20,000 GL261 or CT-2A cells in $5 \mu$ of RPMI medium using a stereotactic frame. This model has been established in our laboratory using neurological symptoms as an indicating endpoint; median survival times are approximately 20 days(18).

On the day of surgical resection, 7 days after intracranial implantation of glioma cell lines (GL261, CT-2A), mice were taken to the MRI suite, and successful tumor implantation was confirmed by T2-weighted volumetric brain scans of the animals. After completion of the MRI scan, the animals were taken into the surgical procedure suite. Throughout the entire procedure, sterile aseptic techniques were used. Mice were anesthetized with 1.5\%-5\% isoflurane and $100 \%$ oxygen using an anesthesia vaporizer and monitored. Upon loss of hind-foot withdrawal and corneal reflex to gentle touch with a cotton swab, the mice were deemed ready for surgery. The fur along the left aspect of the cranium was shaved, and the mice were placed in a stereotactic frame with an adapted nose cone for continuous inhaled anesthetic delivery throughout the procedure. The cranium and future incision site were cleaned with $10 \%$ povidone-iodine (Medline, 53329-945-09) x3, the region around the surgical site was draped, and the operating microscope was brought into the field (Leica Microsystems, 6x scope). Subcutaneous lidocaine was injected along the planned incision. A \#15 blade scalpel (Futura, SMS215) was used to make a linear cranial-caudal incision through the skin to the cranium, and the skin flaps were retracted using 5-0 polypropylene sutures (Ethicon). The periosteum was scraped using a \#4 Pennfield retractor. Using an 
electric handheld drill with a $1.6 \mathrm{~mm}$ carbide round bur (Roboz Surgical Instruments, RS-6280C-5), copious irrigation with sterile saline, and suctioning, a $4 \mathrm{~mm}$ craniotomy was generated overlying the point of intracranial injection. Bony fragments were removed using micro forceps. The dura was incised using micro-scissors, and hemostasis was achieved by copious irrigation and oxidized regenerated cellulose matrix (Surgicel). A corticectomy was performed using a medium \#5 Fukushima suction tip and tissue retraction with \#4 Pennfield retractor. The gross tumor was visualized, and using a combination of suction, retraction, and microdissection, the tumor was de-bulked until no visible gross tumor remained. The surgical cavity was irrigated and inspected for visible tumor. Upon satisfactory visualization of only normal brain, the surgical cavity was lined with oxidized regenerated cellulose matrix (Surgicel). A larger piece of cellulose matrix was placed over the overlying dura. The retracting sutures were removed, and the skin was approximated using a running simple stitch using 6-0 polypropylene suture (Ethicon). The microscope was removed from the field, and the mouse was taken out of the stereotactic frame. Sterile normal saline $(1 \mathrm{~mL})$ was injected subcutaneously for hydration, and one dose of $0.1 \mathrm{mg} / \mathrm{kg}$ buprenorphine was given for analgesia before recovery. The mice were treated with either PBS or dexamethasone (Sigma-Aldrich, $4 \mu \mathrm{g}$ ) intraperitoneally. The mice were then placed on a heating pad and allowed to emerge from anesthesia. A second dose of buprenorphine was given 4-6 hours later on the day of surgery and another the following morning. After the third dose, buprenorphine was given up to three times daily PRN if the animals appeared to be in pain. Mice received $1 \mathrm{ml}$ sterile normal saline daily for 4 days post operatively to prevent dehydration. Animals were provided with prophylactic antibiotics (neomycin, 500 $\mu \mathrm{g} / \mathrm{ml}$ ) and analgesics (ibuprofen, $200 \mu \mathrm{g} / \mathrm{ml}$ ) added to their drinking water. Dexamethasone (Sigma-Aldrich, $4 \mu \mathrm{g}$ ) or PBS was administered intraperitoneally daily in the afternoon for the duration of the experiments.

\section{MRI}

Mice were imaged at 7 days post-intracranial implantation and $24 \mathrm{~h}$ and 14 days post-tumor resection. MRI acquisitions were carried out on a 7 T MRI scanner (Bruker BioSpec 70/20 USR; Billerica, MA) using a 23-mm volume coil setup. Animals were anesthetized with an isoflurane/oxygen mixture (1-3\%, VetFlo System, Kent Scientific) throughout the scan acquisition, with respiration and body temperature monitored via a physiological monitoring system (SA Instruments, Stoney Brook, NY). The animal's head was consistently positioned within the 23-mm volume coil to ensure that the entire brain region was being scanned and that slice-to-slice comparisons could be made between the two time points. The imaging protocol utilized was a multi-slice acquisition (axial) fast-spin echo (FSE) sequence (T2TURBORARE, Paravision 6.0) to provide structural information of the tumor volume and location. The T2-weighted FSE sequence was run with the following parameters: FOV $=1.8$ $\times 1.8 \mathrm{~cm}$, slice thickness of $0.5 \mathrm{~mm}$, matrix size $180 \times 180, \mathrm{TE}=50 \mathrm{~ms}$ (Echo Spacing $=7.0$ and $\mathrm{ETL}=16), \mathrm{TR}=4550 \mathrm{~ms}$, and $\mathrm{SA}=6$ with $\mathrm{TA}=5 \mathrm{~min}$ per animal.

\section{Edema Scoring}

From the volumetric T2-weighted axial MRI slices obtained for each mouse, the overall gross tumor was identified and highlighted using BrainLab 3.0 software. The visible T2 hyper-intensities outside of the tumor volume were identified as the surrounding vasogenic 
edema and scored on a scale of $0-3$ to correlate tumor volume with edema volume.

Importantly, the individual analyzing each MRI image was blinded to the treatment the mice received until after the edema was analyzed for all murine subjects.

\section{Patient Sample Volumetric}

BrainLab 3.0 software was utilized to analyze the axial MRI slices of each mouse. The overall tumor area was outlined manually for each image in a treatment-blinded manner.

\section{Flow Cytometry}

Antibody staining and flow cytometry were performed as previously described by our laboratory(19,20). Briefly, at the designated time point, 1 week after surgical resection, blood was collected in EDTA tubes form terminal bleeds, and the spleen, bone marrow, tumor and non-tumor tissue harvested and mechanically dissociated using $40 \mu \mathrm{M}$ cell strainers. Each sample was then stained for live/dead cells using a Fixable Blue Dead Cell Stain Kit (Thermo Fisher Scientific, Catalog \# L23105) and blocked using Fc Receptor block (Miltenyi Biotec 130-092-575). Next, samples were split into two parts for myeloid and lymphoid panel staining. The myeloid panel included: live/dead UV, CD45, CD11b, CD11C, IA/E, CD103, Ly6G, Ly6C, CD68, and Ki67, and the lymphoid panel included: live/dead UV, CD45, CD3, CD4, CD8, PD1, NK1.1, CD25, CD69, and FoxP3. Antibodies were obtained from Biolegend (San Diego, CA) for analysis of mouse immune profile and included fluorophore-conjugated anti-Ly6C (Clone HK1.4, Catalog \# 128024), anti-Ly6G (Clone A8, Catalog \# 127618), anti-CD11b (Clone M1/70, Catalog \# 101212), anti-CD68 (Clone FA-11, Catalog \# 137024), anti-I-A/I-E (Clone M5/114.15.2, Catalog \# 107606), anti-CD11c (Clone N418, Catalog \# 117330), anti-CD3 (Clone 145-2C11, Catalog \# 100330), anti-CD4 (Clone GK1.5, Catalog \# 100422), anti-CD8 (Clone 53-6.7, Catalog \# 100712), anti-NK1.1 (Clone PK136, Catalog \# 108741), anti-CD45 (Clone 30-F11, Catalog \# 103132), anti-Ki-67 (Clone 16A8, Catalog \# 652404). Gating for MDSCs was performed using FlowJo v10, and M-MDSCs were identified by (Singlets/Live/CD45+/CD11b+/ CD68-/IAIE-/Ly6G-/LyC+) and G-MDSCs by (Singlets/Live/CD45+/CD11b+/CD68-/ IAIE-/Ly6C-/Ly6G+). T cells were identified by (Singlets/Live/CD45+/CD3+/NK1.1-). CD4+ T cells were identified by (Singlets/Live/CD45+/CD3+/NK1.1-/CD4+/CD8-), CD8+ T cells by (Singlets/Live/CD45+/CD3+/NK1.1-/CD4-/CD8+), NK cells by (Singlets/Live/ CD45+/NK1.1+), T regulatory cells by (Singlets/Live/CD45+/CD3+/CD4+/FoxP3+), and macrophages by (Singlets/Live/CD45+/CD11b+/CD68+/IAIE+). CD45+ cells are graphed as a percentage of live cells, while all other populations are graphed as percentage of live/ single/CD45+ cells.

\section{Study approval}

Patient data analysis was done via the Rose Ella Burkhardt Brain Tumor and NeuroOncology Center at the Cleveland Clinic in accordance with IRB 2559.

\section{Statistics}

GraphPad Prism and R (version 4.0.2) were used. Times to events (progression or death) were modeled using Cox proportional hazards models. Kaplan Meier survival curve 
differences were assessed by log-rank tests. The $\mathrm{R}$ package survival was used to compute $\log$ rank tests and to fit Cox models in R; the R package survminer was used to form KapanMeier plots and forest plots of hazard ratio estimates. Automated exhaustive Cox model space searches were performed using the R package glmulti with Akaike's Information Criterion (AIC) as the model selection criterion. Relative to the Bayesian Information Criterion (BIC), AIC tends to select larger models; both metrics strike balances between desires to increase goodness-of-fit and desires to fit fewer model parameters. Other data (e.g. flow cytometry data) was analyzed using the R packages ggplot2 and ggpubr, which integrate basic statistical tests with plotting. P-values were considered statistically significant at $* \mathrm{p}<0.05, * * \mathrm{p}<0.01$, and $* * * \mathrm{p}<0.001$.

\section{Results}

\section{Establishment of a clinically relevant mouse model of GBM surgical resection}

Given the inherent difficulties of studying the singular effects of surgical resection or steroid treatment in patients with GBM, we sought to develop a murine model of GBM surgical resection with a translationally relevant steroid dose. The steroid-treated mice received $4 \mu \mathrm{g}$ dexamethasone daily, which corresponds by weight to a dosage of $16 \mathrm{mg}$ of dexamethasone per day for an $80 \mathrm{~kg}$ patient. In this initial proof-of-concept investigation, we first intracranially injected GL261 cells at day 0 and waited 7 days before surgically resecting the tumor or performing a mock resection procedure, in which a corticectomy was performed after opening the dura and separating white matter tracks overlying the tumor without removing the tumor. The mock resection cohort was developed to normalize for systemic murine responses to injury or violation of the dura/cranial cortical surface (Fig. 1A)(21). Importantly, time under anesthesia, blood loss, and surgical procedure time did not differ significantly between the two cohorts: the mean time of the surgical procedure was $\sim 20$ minutes in both cohorts. MRI images were obtained for each mouse prior to surgical intervention, after resection, and 7 days post-procedure, when recurrent tumor spreading throughout the brain was typically observed, usually either along the surface of the resection cavity or along white matter tracts (Fig. 1B). Volumetric analysis of post-resection animals, in both the PBS and dexamethasone $4 \mu \mathrm{g}$ daily cohorts, demonstrated successful tumor debulking and resultant decreases in recurrent tumor volumes at 7 days post-surgery (Fig. 1C, D, Supplemental Figure 1A). Utilizing this model, surgical resections compared to mock surgical corticectomies demonstrated a median survival benefit of 4 days (Fig. 1E). As expected, administration of therapeutic levels of dexamethasone (4 $\mu \mathrm{g}$ daily per $20 \mathrm{~g}$ animal) did not alter survival in either GL261 or CT-2A tumor-bearing mice with or without surgical resection of the tumors (Fig. 1F, Supplemental Fig. 1B, C). Importantly, MRI analysis of dexamethasone-treated mice showed the expected reduction in tumor-induced vasogenic edema (Fig. 1G, H). These data demonstrate a clinically appropriate model of surgical resection and steroid treatment in mice that can be utilized to investigate immunological changes due to initial upfront GBM therapies.

\section{Surgical resection causes systemic immune suppression}

After development of the surgical resection model, we sought to utilize it to determine whether there are differences in local and systemic immune responses related to initial 
treatment. For studies relating to the immunobiology of treatment, we chose a time point of 7 days post-resection to allow for the effects of dexamethasone treatment to develop and for temporal resolution of the presumed initial injury and healing response to surgery. This also permitted analysis of the recurrent tumor at that time. Based on previous studies of CNS injury response(21), this time point should show the lasting immunologic effects separate from the acute injury response. Thus, mice in an initial cohort were implanted with tumors, that were allowed to grow before being resected as described above, and 7 days postresection, an MRI was performed. Flow cytometry for lymphoid (T cells, CD4+ T cells, CD8+ T cells, natural killer (NK) cells, T regulatory cells) and myeloid (myeloid-derived suppressor cells (MDSCs), macrophages, dendritic cells, and monocytes) cells was performed on samples of blood, spleen, gross recurrent tumor, non-tumor cortex within the recurrent tumor-bearing hemisphere, and bone marrow. There were no major differences noted between the percentage of CD45+ live cells present among any of the treatment conditions and organs except in the spleen and tumor after surgical resection of the brain tumor (Supplemental Fig. 2). Initial analysis of immunophenotypic changes in GL261bearing mice revealed that the immune-suppressive granulocytic MDSCs (G-MDSCs) were proportionally increased in the blood of mice in response to dexamethasone treatment alone as well as mildly increased due to surgical resection (Supplemental Figs. 3, 4). Immune suppressive G-MDSCs were also increased in the recurrent tumors after resection and/or steroid treatment compared to mock resected animals (Supplemental Fig. 3). More strikingly, we observed a reduction in circulating T cells, including CD8+ T cells, following surgical resection alone, with a corresponding increase in $\mathrm{CD} 8+\mathrm{T}$ cells in the bone marrow (Fig. 2A-B). These observations were repeated in the CT-2A resection model, and a similar decrease in CD8+ T cells in the blood was observed due to surgical resection, with a corresponding increase in CD8+ T cells in the bone marrow (Fig. 2C-D, Supplemental Figs. 5-7). To determine how $\mathrm{T}$ cell trafficking is altered due to surgical intervention we next performed high dimensional flow cytometry with a focus on CD4 and CD8 T cells staining for the following markers: CD45, CD3, CD4, CD8, CCR7, CD62L, CXCR3, CXCR4, LFA1, PD-1(Supplemental Fig. 8). Accompanying our findings of reduced CD8 T cell counts in the blood, we also observed their reduced expression of CCR7 (Fig 2E). These observations indicate that removing the tumor was followed by a reduction in the number of circulating lymphocytes (Fig. 2F), and we next sought to determine whether tumor volume directly affected the levels of circulating $\mathrm{T}$ cells.

\section{Murine tumor volume negatively correlates with circulating $T$ cell numbers in the absence of steroid treatment}

To further understand how tumor volume and surgical resection might impact systemic immune parameters, we performed a volumetric analysis of the MRIs available at day 7 post-resection, the same day that flow cytometry was performed. Across a larger cohort of experimental mice, analysis of the bone marrow in the mock PBS, resection PBS, mock dexamethasone, and resection dexamethasone groups revealed a linear relationship between recurrent tumor volume and the quantity of T cells in the bone marrow (Fig. 3A-D). Of note, the number of CD8+ T cells was significantly higher in all groups compared to the combination of surgical resection and dexamethasone treatment, highlighting the combined impact of the two therapies. Further analysis of the blood lymphocyte counts revealed an 
inverse correlation between the number of $\mathrm{T}$ cells in the circulation and tumor volume (Fig.

3E-H). This inverse correlation was readily apparent when surgical resection was not performed, and similar trends were also present in the resection PBS group (Fig. 3E-H). Indepth analysis of recurrent tumor volume and its correlation with myeloid cell populations and lymphocytes within the spleen, blood, bone marrow, recurrent tumor and non-tumor cortex did not identify any other consistent correlations with recurrent tumor volume (Supplemental Figs. 9-13). Thus, our murine model suggests that $\mathrm{T}$ cells in the blood and marrow decrease and increase, respectively, with tumor volume, and that these relationships vanish when surgical resection and steroid treatment are both applied.

\section{Steroid-naïve GBM patients exhibit an inverse correlation between peripheral blood lymphocyte counts and progression-free and overall survival}

To determine if relationships identified in our mouse model also existed in human patients with GBM, we assessed a clinical cohort of GBM patients who had an MRI as well as a complete blood count with differential (CBC w/ diff) prior to surgical resection. After analysis of patient records from a cohort of $>400$ GBM patients, we identified 95 GBM patients who met these criteria (Table 1). The demographics of the cohort utilized were analogous to the larger population of newly diagnosed GBM patients taken to surgery within our institution who did not meet the above criteria. Within the cohort of 95 newly diagnosed GBM patients, 61 patients had a CBC count prior to receiving any steroid treatment, while the other 34 patients had already received or were currently on steroids at the time of the $\mathrm{CBC}$ w/ diff, all prior to surgery. Overall survival dependencies on Stupp protocol, Tumor laterality, Resection type, Post operation MRI contrasted tumor volume, and age were all as expected and significant by log-rank tests (Supplemental Fig. 14 A-F). Comparison of those patients who received steroids prior to surgery $(\mathrm{n}=34)$ and those who did not receive steroids prior to surgery $(\mathrm{n}=61)$ demonstrated the expected reduction in circulating lymphocytes, with no effect on the monocyte populations (Fig. 4A, Supplemental Fig. 15A). Additionally, we see that even with steroid treatment patients there is a trend toward surgical resection reducing peripheral blood lymphocyte counts, as compared to biopsy (Supplemental Fig. 15B). Tumor volumes were then calculated from the MRIs corresponding to the time of $\mathrm{CBC}$ w/ diff, and we found that steroid-naïve patients demonstrated an inverse correlation between tumor volume and both lymphocyte percentage and absolute count by $\mathrm{CBC}$ w/ diff (Fig. 4B, Supplemental Fig. 15C-D). In contrast, steroid-treated patients did not exhibit the same correlations (Fig. 4C, Supplemental Fig. 15C-D). Univariate and multivariate Cox model analyses of the relationship between peripheral lymphocyte counts and progressionfree survival (PFS) and overall survival (OS) were performed. In these analyses the PFS and OS were analyzed with the clinical variables: \%Lymphocytes pre-surgery, Absolute Lymphocytes pre-surgery, Steroid treatment pre-surgery, Tumor Volume, Year Diagnosed, Age, Sex, Type of resection, Biopsy vs Resection, Tumor Laterality, KPS, 1p, 19q, ki67, and EGFR Amplification status. Within these analyses we have excluded the completion of Stupp protocol based on its dependence on other variables in the model such as type of resection, tumor laterality, biopsy vs resection, lymphocyte count, and residual tumor volume which are all already included in the models. Multivariable Cox ProportionalHazards modeling was performed on these variables using the R package glmulti with automated model selection set to identify the top 5 models based on Akaike's Information 
Criterion (AIC), exhaustively searching the entire model space. Of these 5 models we display the top model for progression free survival (Fig. 4D) and Overall Survival (Fig. 4E), both of which include the absolute lymphocyte count at diagnosis; absolute lymphocyte count at diagnosis was also present in the four runner up models (Supplemental Figures 16, 17), which have similar AIC values and are thus close competitors. Importantly, other variables such as Biopsy vs resection, age, sex, 1p19q status, and tumor laterality are present in the progression free survival model and are known to affect GBM prognosis (Fig. 4D, Supplemental Fig. 16A-D). Of note, steroid treatment pre-op was also identified as a variable within the models of overall survival, and this was recently identified in another cohort of 88 patients with GBM(15) (Fig. 4E, Supplemental Fig. 17A-D). After identifying that the absolute lymphocyte count is correlated with PFS and OS we sought to understand if the lymphocyte count is consistent over time in GBM patients based on their initial CBC/ diff. In steroid-naïve patients, we identified that initial lymphocyte counts correlate with counts at recurrence (Fig. 4F) and in steroid naïve patients, with PFS and OS, but this is lost in steroid treated patients (Supplemental Fig. 18A-B).

\section{Conclusions/Discussion}

As immunotherapies for GBM evolve, the context in which they will be administered, and more specifically the impact of existing standard-of-care paradigms, must be considered. In particular, interactions with surgical resection, steroids, chemotherapy, and radiation, must be assessed, as must the importance of their timings relative to administration of immunemodulating agents. This was highlighted in recent findings that dexamethasone treatment negatively impacts overall survival in the context of immunotherapy $(15,16)$. This is in contrast to our findings in immunotherapy naïve patients that dexamethasone prior to surgery was beneficial, in multivariate analyses (Figure4E). The studies performed here combined with recent clinical trial results(15) indicate that dexamethasone can be beneficial in the standard-of-care chemotherapy setting but not in the case of immunotherapy. Our studies expand on these observations first by identifying and clinically validating a model of how standard of care impacts the immune response and, secondly, by identifying a novel association between tumor size and reduced circulating lymphocytes.

Systemic reduction in T cells following surgical resection and steroid treatment appear to correlate with a reduction in T cell CCR7 expression. This may be relevant as CCR7 loss has been shown to cause T cells to migrate to IL-15 rich niches, such as the bone marrow. Such migration could be less favorable for an anti-tumor immune response when compared to CCR7 high T cells migrating to IL-7 niches(22). The factor or set of factors causing T cells to lose CCR7 following surgical resection require more in depth studies of patient cohorts. In GBM, we see these findings as immediately relevant, particularly in light of recent clinical trial findings and the need to enhance immunotherapies in $\operatorname{GBM}(16)$. Lately, immunotherapy in GBM failed in the CheckMate 143 clinical trial(23), where recurrent GBM patients who had previously failed standard-of-care therapy were evaluated for their response to anti-PD1 immune checkpoint therapy. Following this trial, a smaller study was initiated with therapy delivered prior to surgical resection (neoadjuvant)(14), and moderate but promising effects were observed. New clinical trials testing neoadjuvant immunotherapy 
are increasingly being attempted. Preclinical modeling of these paradigms is critical, as it will generate hypotheses regarding how surgery, steroids, and the immune system interact.

Using mouse GBM models, we determined that surgical resection alone leads to a reduction in circulating T cells, in addition to the known impact of steroid treatment(24-26), which is also being revisited in terms of its clinical use. Many groups have shown that $\mathrm{T}$ cell abundance is one of multiple factors influencing immunotherapy response. Our murine mock-resection model enables studies of cohorts of mice with similar time of surgery, time under anesthesia, blood loss, dural penetration, and disruption of native cortex. This allowed us to conclude that regardless of the initial factor causing a reduction in lymphocytes, the simple act of tumor debulking also causes a reduction of $\mathrm{T}$ cells in the circulation. Here we focused primarily on implantable models because the use of genetically engineered mouse models of glioma would be more difficult to resect safely in a mouse, but the validation in GBM patients and two different implantable models makes us confident that this phenomenon is prevalent. Furthermore, immune induced sequestration of $\mathrm{T}$ cells to the bone marrow was previously demonstrated to be a fairly universal accompaniment to intracranially situated tumors, regardless of the type of tumor implanted in the brain, be it glioma, melanoma, breast, or lung cancer cell lines(3).

Strengthening our overall findings in the mouse model, evaluation of a cohort of GBM patients verified that surgical resection, as compared to biopsy, reduces peripheral blood lymphocyte counts, even when steroids are present (Supplemental Fig. 15B). Furthermore, T cell sequestration in the bone marrow in the setting of newly diagnosed intracranial tumors has been previously described $(3,27)$. We observe the same accumulation of $\mathrm{T}$ cells in the bone marrow in murine models of surgically resected, recurrent tumors and additionally that this accumulation varies with tumor volume. As mice studied are syngeneic, decreasing lymphocyte counts is unlikely to be due to differences in host immune constitution. We also observe an inverse relation between peripheral blood lymphocyte counts and tumor volume in steroid-naïve human patients. In these patients decreased pre-operative lymphocyte counts correlated with worse progression-free- and overall survival in multivariable Cox models. Additionally, those patients who had steroid treatment prior to surgical resection at the time of the CBC w/diff had worse overall survival, which could be due to the severity of disease but could also be due to a reduced ability to respond to the tumor.

Critically, contrasted pre-operative tumor volume not being a significant risk factor in uniand multi-variate models of progression-free- and overall survival suggests that mechanistically, preoperative lymphocyte counts are not merely surrogate measures of tumor volume/stage but instead may reflect a patient's underlying ability to mount an immune response to GBM. Similarly, steroid use dropping out of significance in comparisons of multivariate hazard ratios of progression free survival suggests that a patient's intrinsic or at least pre-operative immune status is a stronger determinant of the risk of disease progression. These models demonstrate the importance of patient lymphocyte counts on outcome, as lymphocyte numbers have a larger impact than the standard treatment protocol (including radiation and concurrent/adjuvant temozolomide treatment) on outcome. This finding is of particular interest because it indicates that those patients who have high lymphocyte counts may not necessarily receive maximal benefit from the current standard- 
of-care treatments but could instead benefit from an immunotherapy-based approach(16). Furthermore, we also noted a significant increase in PD-1 expression on CD8 T cells in response to resection, and this was further increased in the bone marrow by a combination of dexamethasone and resection, which aligns with $\mathrm{T}$ cells homing to the bone marrow and being more exhausted. Studies such as this may indicate that these patients would derive greater benefit from immune-activating strategies, but only when dexamethasone is not used in combination with surgical resection.

Peripheral lymphocyte counts at diagnosis, prior to steroid administration, may reveal which patients are more likely to benefit from immuno-therapies. Patients with higher baseline lymphocyte counts might be more likely to benefit from neoadjuvant checkpoint blockade and patients with lower counts might benefit from receiving $\mathrm{T}$ cell-activating or -mobilizing therapies. A more complete understanding of a patient's baseline immune status and the temporal effects of administration of therapies on the immune system is needed to enable separations of patients into subgroups more likely to benefit from a given immunotherapy. Developing such initial assessments will likely be critical to the success of GBM immunotherapies in clinical trials.

\section{Supplementary Material}

Refer to Web version on PubMed Central for supplementary material.

\section{Acknowledgments}

We thank the members of the Lathia laboratory for insightful discussion and constructive comments on the manuscript. We thank the Lerner Research Institute Flow Cytometry Core for their assistance. We thank Amanda Mendelsohn and the Center for Medical Art and Photography at the Cleveland Clinic for providing illustrations.

Funding

This work was funded by an NIH grant (R01 NS109742 to JL and MAV, F31 NS101771 to TA, F32 CA243314 to DB) the Sontag Foundation (JL, PF), Cleveland Clinic Research Program Committees grant to (BO), Blast GBM (JL and MV), the Cleveland Clinic VeloSano Bike Race (JL and MV), B*CURED (JL and MV), the Case Comprehensive Cancer Center (JL and MV), and the Cleveland Clinic Brain Tumor Research and Therapeutic Development Research Center of Excellence (MA and JL).

\section{References}

1. Stupp R, Taillibert S, Kanner A, Read W, Steinberg D, Lhermitte B, et al. Effect of Tumor-Treating Fields Plus Maintenance Temozolomide vs Maintenance Temozolomide Alone on Survival in Patients With Glioblastoma: A Randomized Clinical Trial. JAMA 2017;318(23):2306-16 doi 10.1001/jama.2017.18718. [PubMed: 29260225]

2. McGranahan T, Therkelsen KE, Ahmad S, Nagpal S. Current State of Immunotherapy for Treatment of Glioblastoma. Curr Treat Options Oncol 2019;20(3):24 doi 10.1007/s11864-019-0619-4. [PubMed: 30790064]

3. Chongsathidkiet P, Jackson C, Koyama S, Loebel F, Cui X, Farber SH, et al. Sequestration of T cells in bone marrow in the setting of glioblastoma and other intracranial tumors. Nat Med 2018 doi 10.1038/s41591-018-0135-2.

4. Lim M, Xia Y, Bettegowda C, Weller M. Current state of immunotherapy for glioblastoma. Nat Rev Clin Oncol 2018;15(7):422-42 doi 10.1038/s41571-018-0003-5. [PubMed: 29643471] 
5. Huang J, Liu F, Liu Z, Tang H, Wu H, Gong Q, et al. Immune Checkpoint in Glioblastoma: Promising and Challenging. Front Pharmacol 2017;8:242 doi 10.3389/fphar.2017.00242. [PubMed: 28536525]

6. Filley AC, Henriquez M, Dey M. Recurrent glioma clinical trial, CheckMate-143: the game is not over yet. Oncotarget 2017;8(53):91779-94 doi 10.18632/oncotarget.21586. [PubMed: 29207684]

7. Heynckes S, Daka K, Franco P, Gaebelein A, Frenking JH, Doria-Medina R, et al. Crosslink between Temozolomide and PD-L1 immune-checkpoint inhibition in glioblastoma multiforme. BMC Cancer 2019;19(1):117 doi 10.1186/s12885-019-5308-y. [PubMed: 30709339]

8. Sengupta S, Marrinan J, Frishman C, Sampath P. Impact of temozolomide on immune response during malignant glioma chemotherapy. Clin Dev Immunol 2012;2012:831090 doi 10.1155/2012/831090. [PubMed: 23133490]

9. Wang S, Yao F, Lu X, Li Q, Su Z, Lee JH, et al. Temozolomide promotes immune escape of GBM cells via upregulating PD-L1. Am J Cancer Res 2019;9(6):1161-71. [PubMed: 31285949]

10. Karachi A, Dastmalchi F, Mitchell DA, Rahman M. Temozolomide for immunomodulation in the treatment of glioblastoma. Neuro Oncol 2018;20(12):1566-72 doi 10.1093/neuonc/noy072. [PubMed: 29733389]

11. Rajani KR, Carlstrom LP, Parney IF, Johnson AJ, Warrington AE, Burns TC. Harnessing Radiation Biology to Augment Immunotherapy for Glioblastoma. Front Oncol 2018;8:656 doi 10.3389/ fonc.2018.00656. [PubMed: 30854331]

12. Kesarwani P, Prabhu A, Kant S, Kumar P, Graham SF, Buelow KL, et al. Tryptophan Metabolism Contributes to Radiation-Induced Immune Checkpoint Reactivation in Glioblastoma. Clin Cancer Res 2018;24(15):3632-43 doi 10.1158/1078-0432.CCR-18-0041. [PubMed: 29691296]

13. Nesseler JP, Schaue D, McBride WH, Lee MH, Kaprealian T, Niclou SP, et al. Irradiation to Improve the Response to Immunotherapeutic Agents in Glioblastomas. Adv Radiat Oncol 2019;4(2):268-82 doi 10.1016/j.adro.2018.11.005. [PubMed: 31011672]

14. Cloughesy TF, Mochizuki AY, Orpilla JR, Hugo W, Lee AH, Davidson TB, et al. Neoadjuvant antiPD-1 immunotherapy promotes a survival benefit with intratumoral and systemic immune responses in recurrent glioblastoma. Nat Med 2019;25(3):477-86 doi 10.1038/s41591-018-0337-7. [PubMed: 30742122]

15. Nayak L, Molinaro AM, Peters KB, Clarke JL, Jordan JT, de Groot JF, et al. Randomized phase II and biomarker study of pembrolizumab plus bevacizumab versus pembrolizumab alone for recurrent glioblastoma patients. Clin Cancer Res 2020 doi 10.1158/1078-0432.CCR-20-2500.

16. Iorgulescu JB, Gokhale PC, Speranza MC, Eschle BK, Poitras MJ, Wilkens MK, et al. Concurrent Dexamethasone Limits the Clinical Benefit of Immune Checkpoint Blockade in Glioblastoma. Clin Cancer Res 2020 doi 10.1158/1078-0432.CCR-20-2291.

17. Oh T, Fakurnejad S, Sayegh ET, Clark AJ, Ivan ME, Sun MZ, et al. Immunocompetent murine models for the study of glioblastoma immunotherapy. J Transl Med 2014;12:107 doi 10.1186/1479-5876-12-107. [PubMed: 24779345]

18. Otvos B, Silver DJ, Mulkearns-Hubert EE, Alvarado AG, Turaga SM, Sorensen MD, et al. Cancer stem cell-secreted macrophage migration inhibitory factor stimulates myeloid derived suppressor cell function and facilitates glioblastoma immune evasion. Stem Cells 2016;34(8):2026-39 doi 10.1002/stem.2393. [PubMed: 27145382]

19. Alban TJ, Bayik D, Otvos B, Rabljenovic A, Leng L, Jia-Shiun L, et al. Glioblastoma MyeloidDerived Suppressor Cell Subsets Express Differential Macrophage Migration Inhibitory Factor Receptor Profiles That Can Be Targeted to Reduce Immune Suppression. Frontiers in Immunology 2020;11(1191) doi 10.3389/fimmu.2020.01191.

20. Bayik D, Zhou Y, Park C, Hong C, Vail D, Silver DJ, et al. Myeloid-derived suppressor cell subsets drive glioblastoma growth in a sex-specific manner. Cancer Discov 2020 doi 10.1158/2159-8290.CD-19-1355.

21. Gadani SP, Walsh JT, Lukens JR, Kipnis J. Dealing with Danger in the CNS: The Response of the Immune System to Injury. Neuron 2015;87(1):47-62 doi 10.1016/j.neuron.2015.05.019. [PubMed: 26139369] 
22. Choi H, Song H, Jung YW. The Roles of CCR7 for the Homing of Memory CD8+ T Cells into Their Survival Niches. Immune Netw 2020;20(3):e20 doi 10.4110/in.2020.20.e20. [PubMed: 32655968]

23. Reardon DA, Brandes AA, Omuro A, Mulholland P, Lim M, Wick A, et al. Effect of Nivolumab vs Bevacizumab in Patients With Recurrent Glioblastoma: The CheckMate 143 Phase 3 Randomized Clinical Trial. JAMA Oncol 2020 doi 10.1001/jamaoncol.2020.1024.

24. Giles AJ, Hutchinson MND, Sonnemann HM, Jung J, Fecci PE, Ratnam NM, et al. Dexamethasone-induced immunosuppression: mechanisms and implications for immunotherapy. J Immunother Cancer 2018;6(1):51 doi 10.1186/s40425-018-0371-5. [PubMed: 29891009]

25. Cenciarini M, Valentino M, Belia S, Sforna L, Rosa P, Ronchetti S, et al. Dexamethasone in Glioblastoma Multiforme Therapy: Mechanisms and Controversies. Front Mol Neurosci 2019;12:65 doi 10.3389/fnmol.2019.00065. [PubMed: 30983966]

26. Pitter KL, Tamagno I, Alikhanyan K, Hosni-Ahmed A, Pattwell SS, Donnola S, et al. Corticosteroids compromise survival in glioblastoma. Brain 2016;139(Pt 5):1458-71 doi 10.1093/ brain/aww046. [PubMed: 27020328]

27. Ayasoufi K, Pfaller CK, Evgin L, Khadka RH, Tritz ZP, Goddery EN, et al. Brain cancer induces systemic immunosuppression through release of non-steroid soluble mediators. Brain 2020 doi 10.1093/brain/awaa343. 


\section{Translational Relevance}

With increasing numbers of immunotherapy clinical trials for glioblastoma (GBM), the context in which immunotherapies are administered and the impact of current treatment regimens must be considered. We examined the contribution of initial steroid and surgical resection treatments using a mouse model and validated findings in a cohort of GBM patients. In our pre-clinical model, we identified surgical resection as an event leading to immune suppression, which is further exacerbated by steroid treatment. We identified an inverse correlation between lymphocyte count and tumor volume prior to steroid treatment that was confirmed in a clinical cohort. Further analysis showed that lymphocyte count prior to surgical resection predicted survival, although this is often overlooked due to the known influence of steroid usage on lymphocyte count. Our findings highlight the impact of surgery and steroid usage on immune suppression in GBM, and this effect should be taken into consideration for future immunotherapy trials. 
A

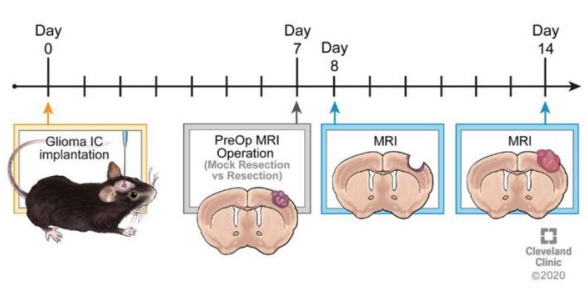

C

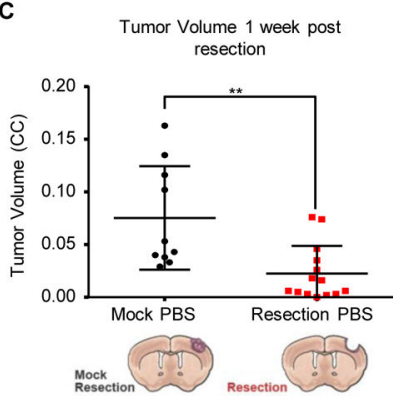

$E$

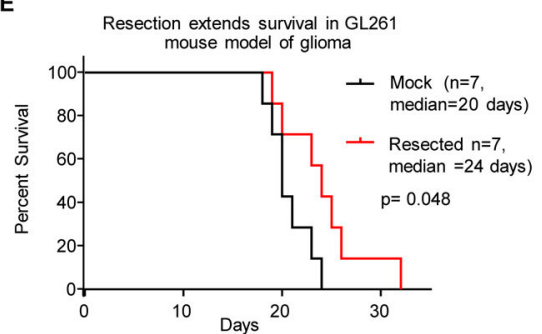

G

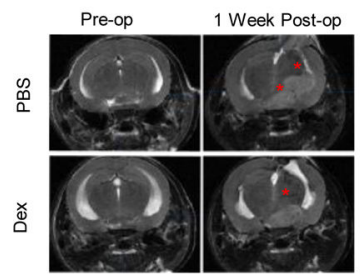

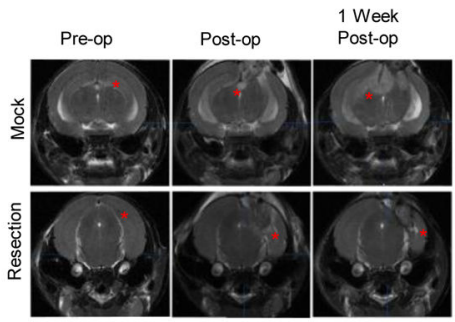

D

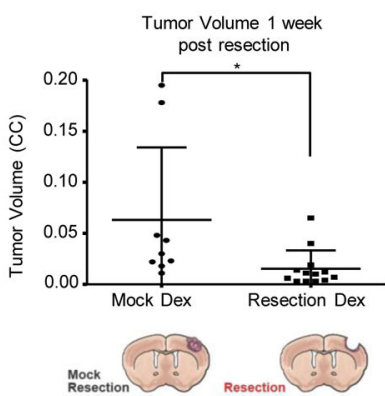

$\mathbf{F}$

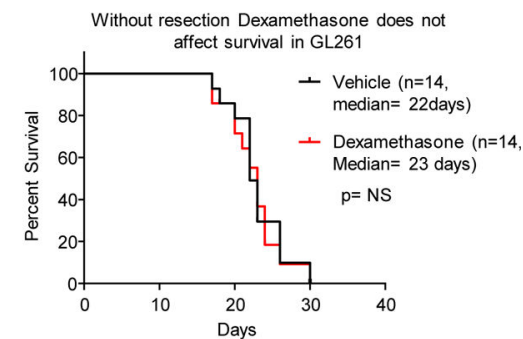

H

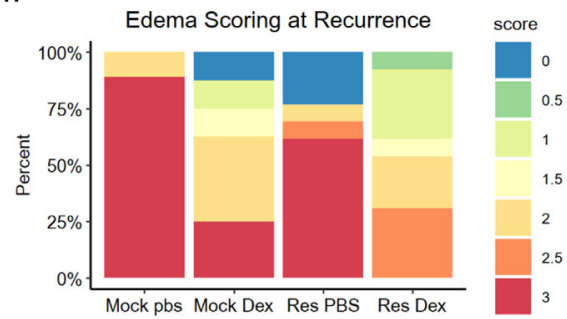

Figure 1- Murine model of resection including dexamethasone treatment extends survival and reduces edema.

To replicate standard-of-care and dexamethasone treatment, a mouse model of resection was initiated as outlined in the diagram, with intracranial implantation of tumor, followed by MRI prior to resection and after resection, and then endpoint MRI along with flow cytometry or survival depending on the experiment (A). Representative MRI images of tumors from the mock resection and resection cohorts pre-, post- and 1 week post-resection (B). Tumor volume was assessed using the BrainLab software suite and graphed as tumor volume $(\mathbf{C}, \mathbf{D})$. A survival study comparing $\mathrm{n}=7$ mice with mock resection and $\mathrm{n}=7$ mice with resection was performed showing a median survival of 20 days for mock resection vs 24 days for resection, with log-rank p-value shown $(\mathbf{E})$. Vehicle- vs dexamethasone (one week at $4 \mu \mathrm{g}$ daily)-treated GL261-bearing mice with no surgical resection were also compared and showed no survival difference due to dexamethasone $(\mathbf{F})$. Representative MRI images of dexamethasone-treated mice and PBS-treated mice pre-op and 1 week post-op 
(G). Edema scoring is graphed as a percentage with $\mathrm{n}=7$ mice per group $(\mathbf{H})$. Student's twotailed t-test was performed for comparisons in panels A, D, E; *p $<0.05$, **p $<0.01$,

$* * * \mathrm{p}<0.001$. Survival curve analysis was performed in GraphPad Prism using log-rank tests (also known as Mantel-Cox tests) for $\mathrm{p}$ values. 


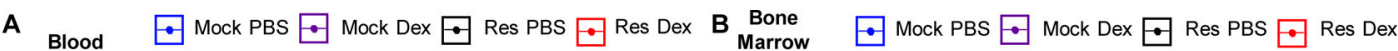
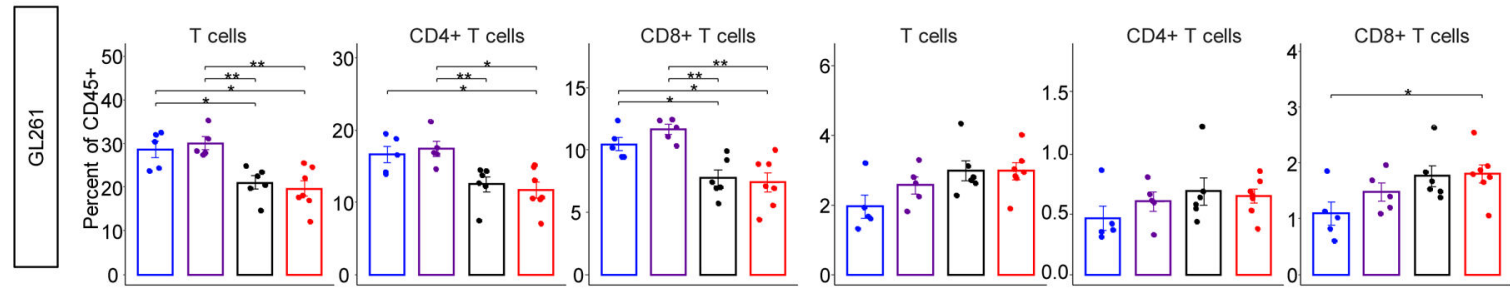

C
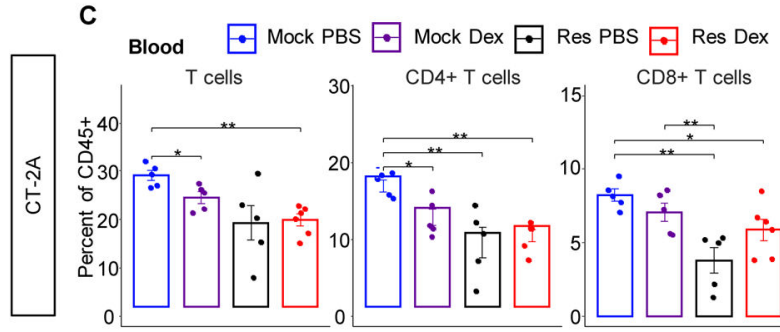

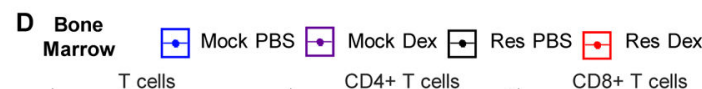
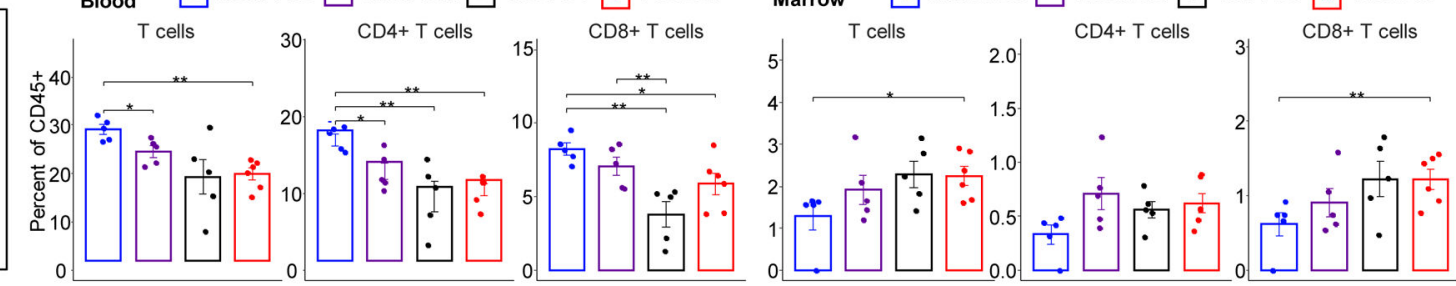

E
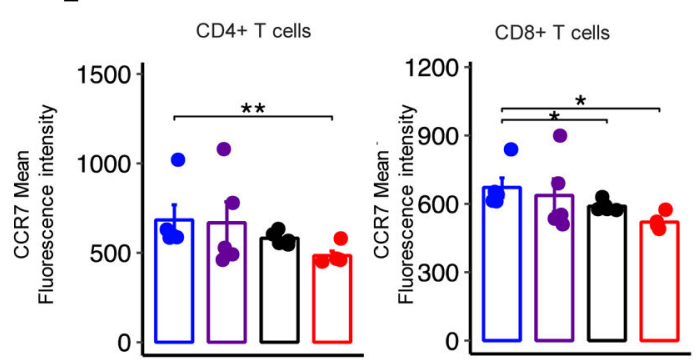

$\mathbf{F}$

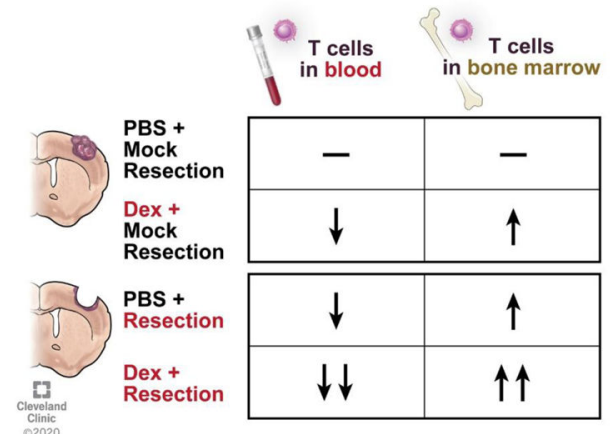

Figure 2- Surgical resection reduces $\mathbf{T}$ cells in the circulation and increases $\mathrm{CD8}+\mathrm{T}$ cells in the bone marrow.

GL261-bearing mice were administered vehicle $(n=7)$ or $4 \mu$ g dexamethasone daily $(n=7)$ for 7 days starting on day 14 post-intracranial injection before being euthanized at day 21 for flow cytometry analysis of the blood and bone marrow (A, B). For the surgical resection model, mock PBS n=5, mock dexamethasone $n=5$, resection PBS n=6, and resection dexamethasone $n=7$. These studies were repeated using the CT-2A model of glioma, and $\mathrm{T}$ cell levels across treatment conditions were analyzed in the blood and bone marrow $(\mathbf{C}, \mathbf{D})$. Mock PBS $n=5$, mock dexamethasone $n=5$, resection PBS $n=5$, resection dexamethasone $\mathrm{n}=6$. CCR7 expression staining for CD4 and CD8 T cells from a resection cohort containing mock PBS $n=5$, mock dexamethasone $n=5$, resection PBS $n=5$, and resection dexamethasone $\mathrm{n}=5$ (E). Summary graphic describing the gross overall $\mathrm{T}$ cell changes in the blood and bone marrow of each treatment $(\mathbf{F})$. Student's two-tailed t-test was performed for the comparisons in panels A, D, E; * $\mathrm{p}<0.05, * * \mathrm{p}<0.01, * * * \mathrm{p}<0.001$. 

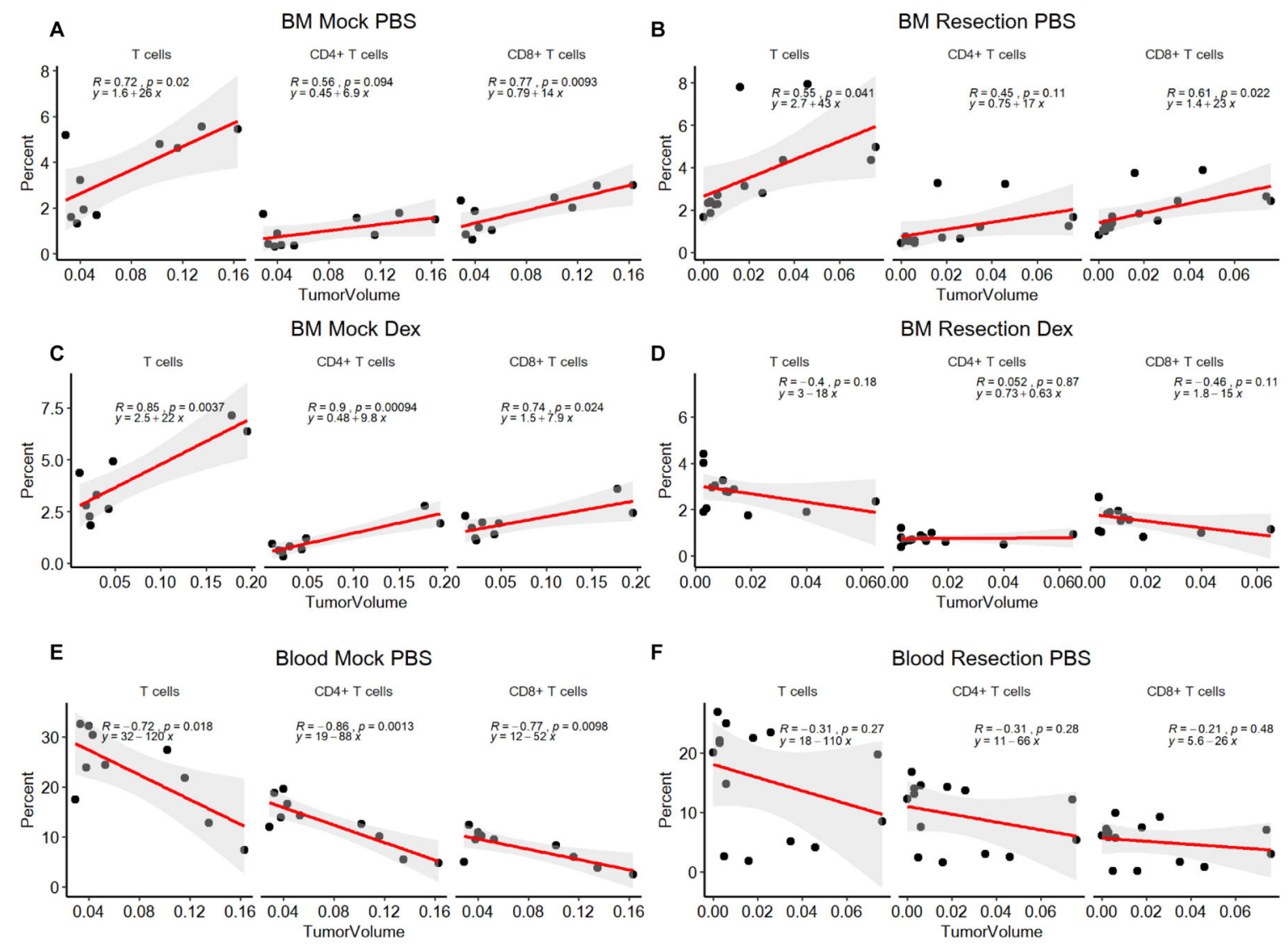

$\mathrm{CD} 4+\mathrm{T}$ cells

$\mathrm{CD} 8+\mathrm{T}$ cells

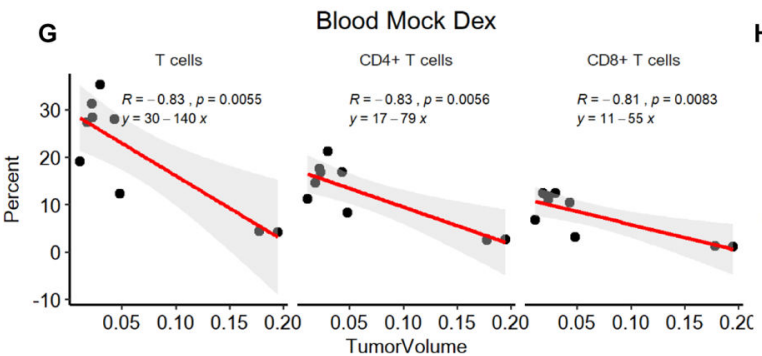

H

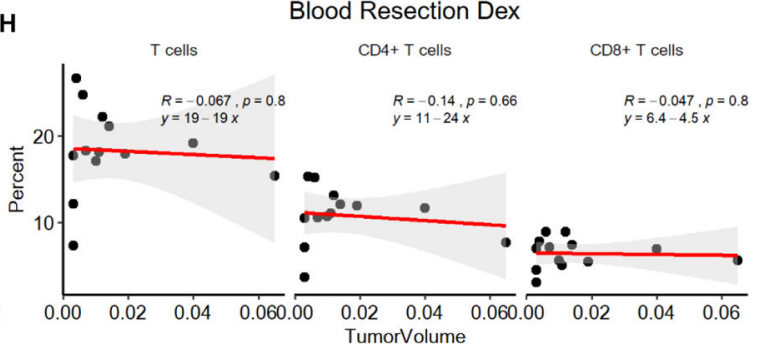

Figure 3- Tumor volume correlates with increased $T$ cells in the bone marrow (BM) and reduced $T$ cells in the blood.

The bone marrow (A-D) and blood (E-H) are graphed as the percentage of T cells, CD4+ and CD8+ of total CD45+ cells, and the tumor volume. $n=10$ for mock PBS, $n=9$ for mock dexamethasone, $n=14$ for resection PBS, and $n=13$ for resection dexamethasone. Pearson correlation coefficients $(\mathrm{R}), \mathrm{p}$ values and estimates of fitted line parameter are shown. 
A

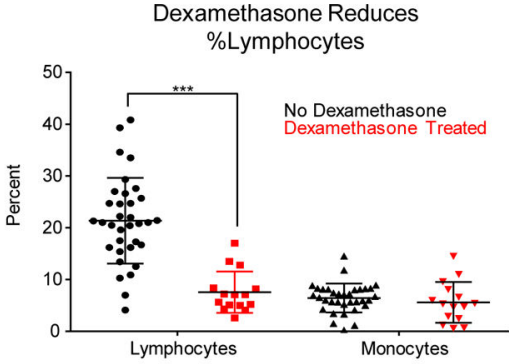

C
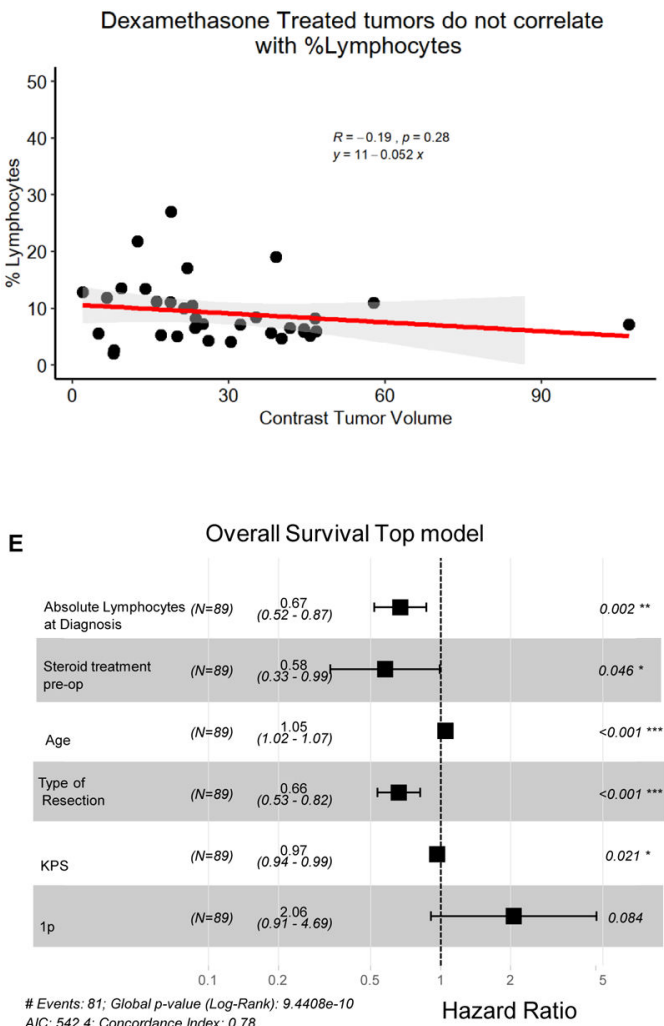
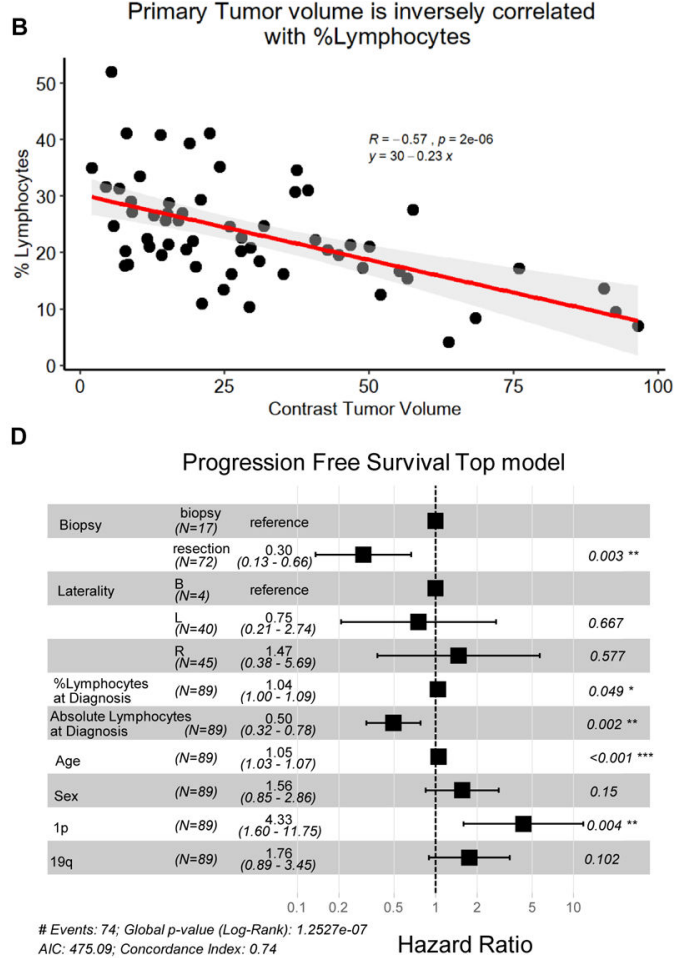

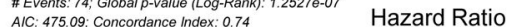

$\mathbf{F}$

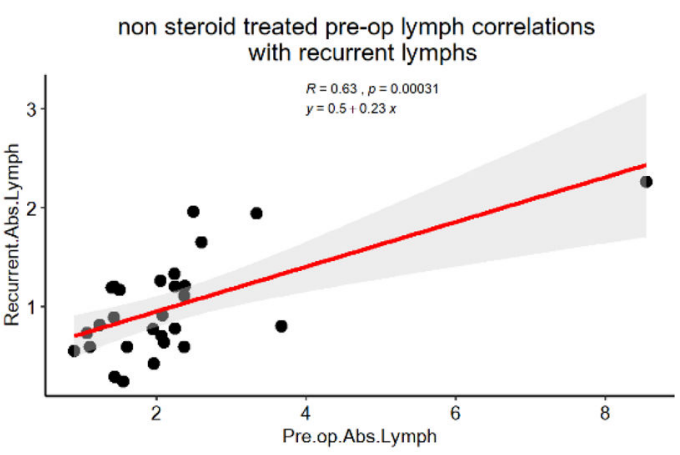

Figure 4- Patient cohort validates that tumor volume negatively correlates with lymphocytes prior to surgery and steroid treatment.

Using a cohort of $n=95$ patients prior to surgical resection, the first $\mathrm{CBC}$ with differential was used to compare \% lymphocytes between dexamethasone-treated- and nondexamethasone-treated patients (A). Percentages of lymphocytes vs tumor volumes (assessed by MRI) of patients without dexamethasone treatment (B). Percentages of lymphocytes vs tumor volumes for those patients treated with dexamethasone prior to surgery who also had a matching MRI at the time of $\mathrm{CBC}$ w/ diff $(\mathbf{C})$. Multivariate Cox proportional hazards model of Progression Free Survival (D). Multivariable Cox proportional hazards model of Overall survival (E). Absolute lymphocyte counts at recurrence and pre-op are correlated for patients not treated with steroids at the time of pre op absolute lymphocyte count $(\mathbf{F})$. The correlation coefficient $(\mathrm{R})$, $\mathrm{p}$ value and fitted line parameter are shown. 


\section{Patient Demographics table.}

Table 1-

\begin{tabular}{|c|c|c|c|c|}
\hline \multirow{2}{*}{ Characteristic } & Total Cohort & $\begin{array}{l}\text { No Steroids Given } \\
\text { Preop Cohort }\end{array}$ & $\begin{array}{l}\text { Steroids Given } \\
\text { Preop Cohort }\end{array}$ & $\begin{array}{l}\text { Significant } \\
\text { Difference }\end{array}$ \\
\hline & \multicolumn{3}{|c|}{$\mathbf{N}(\%)$ or Median Value (IQR) } & p value \\
\hline Total Patients & 95 & $61(64.21 \%)$ & $34(35.79 \%)$ & \\
\hline Age at Diagnosis & $\begin{array}{l}63.72(54.16- \\
70.69)\end{array}$ & $65.16(55.25-72.65)$ & $59.45(51.84-67.51)$ & NS \\
\hline Male Sex & $68(71.58 \%)$ & $41(67.21 \%)$ & $27(79.41 \%)$ & NS \\
\hline Karnofsky Performance Status & $80(80-90)$ & $80(80-90)$ & $90(75-90)$ & NS \\
\hline \multicolumn{5}{|l|}{ Ethnicity } \\
\hline Non-Hispanic White & $86(90.53 \%)$ & $56(91.80 \%)$ & $30(88.23 \%)$ & NS \\
\hline Laterality & & & & NS overall \\
\hline Left & $41(43.16 \%)$ & $25(40.98 \%)$ & $16(47.06 \%)$ & \\
\hline Right & $50(52.63 \%)$ & $32(52.46 \%)$ & $18(52.94 \%)$ & \\
\hline Bilateral & $4(4.21 \%)$ & $4(6.56 \%)$ & $0(0 \%)$ & \\
\hline Lobe & & & & NS overall \\
\hline Deep & $8(8.42 \%)$ & $5(8.20 \%)$ & $3(8.82 \%)$ & \\
\hline Multiple & $18(18.95 \%)$ & $10(16.39 \%)$ & $8(23.53 \%)$ & \\
\hline \multicolumn{5}{|l|}{ Molecular Markers (if known of cohort) } \\
\hline 1p Loss & $11(11.58 \%)$ & $6(9.84 \%)$ & $5(14.71 \%)$ & NS \\
\hline 19q Loss & $13(13.68 \%)$ & $9(14.75 \%)$ & $4(11.76 \%)$ & NS \\
\hline IDH Mutated & $2(2.10 \%)$ & $1(1.64 \%)$ & $1(2.94 \%)$ & NS \\
\hline MGMT Methylated & $13(13.68 \%)$ & $7(11.48 \%)$ & $6(17.68 \%)$ & NS \\
\hline EGFR Amplification & $40(42.11 \%)$ & $26(42.62 \%)$ & $14(41.18 \%)$ & NS \\
\hline Ki-67 & $25 \%(15-50)$ & $27.5 \%(15.5-50)$ & $20 \%(15-50)$ & NS \\
\hline Initial Surgical Management & & & & NS overall \\
\hline Biopsy only & $20(21.05 \%)$ & $13(21.31 \%)$ & $7(20.59 \%)$ & \\
\hline Laser Ablation & $2(2.11 \%)$ & $1(1.64 \%)$ & $1(2.94 \%)$ & \\
\hline Surgical Resection & $70(73.68 \%)$ & $47(77.05 \%)$ & $26(76.47 \%)$ & \\
\hline GTR & $35(36.84 \%)$ & $22(36.06 \%)$ & $13(38.24 \%)$ & \\
\hline NTR & $16(16.67 \%)$ & $13(21.31 \%)$ & $3(8.82 \%)$ & \\
\hline STR & $19(20.00 \%)$ & $12(19.67 \%)$ & $7(20.59 \%)$ & \\
\hline \multicolumn{5}{|l|}{ Adjuvant Therapy } \\
\hline Stupp Protocol Completed & $70(73.68 \%)$ & $41(67.21 \%)$ & $29(85.29 \%)$ & 0.088 \\
\hline $\begin{array}{l}\text { Volume of Contrast-Enhancing Tumor, preop } \\
\text { (cc) }\end{array}$ & $\begin{array}{l}23.75(14.01- \\
41.77)\end{array}$ & $24.20(13.33-43.82)$ & $23.67(15.67-40.57)$ & NS \\
\hline Resection only, preop (cc) & & $29.28(14.12-48.94)$ & $24.96(15.67-40.57)$ & NS \\
\hline Biopsy only, preop (cc) & & $17.69(10.14-21.02)$ & $19.01(17.08-44.51)$ & NS \\
\hline LITT only, preop (cc) & & 5.79 & 5.06 & \\
\hline
\end{tabular}

Clin Cancer Res. Author manuscript; available in PMC 2021 October 01. 


\begin{tabular}{|c|c|c|c|c|}
\hline \multirow{2}{*}{ Characteristic } & Total Cohort & $\begin{array}{l}\text { No Steroids Given } \\
\text { Preop Cohort }\end{array}$ & $\begin{array}{l}\text { Steroids Given } \\
\text { Preop Cohort }\end{array}$ & \multirow{2}{*}{$\begin{array}{c}\begin{array}{c}\text { Significan } \\
\text { Difference }\end{array} \\
\text { p value }\end{array}$} \\
\hline & \multicolumn{3}{|c|}{$\mathbf{N}(\%)$ or Median Value (IQR) } & \\
\hline Volume of FLAIR Abnormality, preop (cc) & $\begin{array}{l}84.71(44.61- \\
127.78)\end{array}$ & $\begin{array}{l}76.94(44.61- \\
129.40)\end{array}$ & $89.78(45.70-124.26)$ & NS \\
\hline $\begin{array}{l}\text { Preop Daily Dexamethasone Dose @ CBC (mg, } \\
\text { if known) }\end{array}$ & $0(0-7)$ & 0 & $16(12-16)$ & $<0.0001$ \\
\hline Resection only, preop, $n=66$ & & 0 & $16(12-16)$ & \\
\hline Biopsy only, preop, $n=20$ & & 0 & $12(6-21)$ & \\
\hline White Blood Cells, preop $\left(x 10^{3} / \mu \mathrm{L}\right), \mathrm{n}=95$ & $8.87(6.44-11.84)$ & $7.57(6.20-9.91)$ & $11.54(8.82-14.85)$ & $<0.0001$ \\
\hline $\begin{array}{l}\text { Percent Lymphocyte Count on CBC, preop, } \\
\text { n=95 }\end{array}$ & $17.60(8.40-25.70)$ & $21.40(17.40-28.95)$ & $7.15(5.43-11.28)$ & $<0.0001$ \\
\hline Resection only, preop, $n=73$ & & $21.3(17.2-28.8)$ & $7.1(5.08-11.28)$ & $<0.0001$ \\
\hline Biopsy only, preop, $n=20$ & & $25.7(17.6-34.3)$ & $8.2(6.3-21.8)$ & 0.009 \\
\hline $\begin{array}{l}\text { Absolute Lymphocyte Count on CBC, preop } \\
\left(\times 10^{3} / \mu \mathrm{L}\right), \mathrm{n}=95\end{array}$ & $1.42(0.90-2.24)$ & $1.73(1.22-2.37)$ & $0.88(0.53-1.21)$ & 0.0002 \\
\hline Resection only, preop & & $1.95(1.20-2.37)$ & $0.875(0.49-1.14)$ & 0.001 \\
\hline Biopsy only, preop & & $1.73(1.19-2.53)$ & $1.23(0.64-2.78)$ & NS \\
\hline Percent Monocyte Count on CBC, preop, $n=95$ & $6.0(3.7-8.0)$ & $6.9(5.3-8.1)$ & $5.8(1.8-6.7)$ & 0.014 \\
\hline $\begin{array}{l}\text { Absolute Monocyte Count on CBC, preop (x10 } 3 \text { / } \\
\mu \mathrm{L}), \mathrm{n}=95\end{array}$ & $0.52(0.34-0.71)$ & $0.52(0.39-0.68)$ & $0.50(0.15-0.85)$ & NS \\
\hline $\begin{array}{l}\text { Volume of Contrast-Enhancing Tumor, post-op } \\
\text { (cc, including biopsies, not including LITT, if } \\
\text { post-op CBC obtained) }\end{array}$ & $0.54(0-7.95)$ & $0.83(0-7.73)$ & $0.35(0-13.70)$ & NS \\
\hline Resection only, post-op, $n=29$ & & $0.50(0-4.51)$ & $0(0-0.54)$ & \\
\hline Biopsy only, post-op, $n=5$ & & $11.98(8.82-18.76)$ & $18.93(15.95-45.03)$ & \\
\hline $\begin{array}{l}\text { Post-op Daily Dexamethasone Dose @ CBC (mg, } \\
\text { if known) }\end{array}$ & $16(8-16)$ & $16(8-16)$ & $16(8-16)$ & NS \\
\hline Resection only, post-op, $n=55$ & & $16(8-16)$ & $16(12-16)$ & NS \\
\hline Biopsy only, post-op, $n=10$ & & $8(5-14)$ & $12(7-22)$ & NS \\
\hline White Blood Cells, post-op $\left(x 10^{3} / \mu \mathrm{L}\right), \mathrm{n}=67$ & $13.27(9.03-16.15)$ & $13.36(8.42-16.03)$ & $12.75(9.62-16.35)$ & NS \\
\hline $\begin{array}{l}\text { Percent Lymphocyte Count on CBC, post-op, } \\
n=67\end{array}$ & $8.2(4.8-14.1)$ & $8.45(5.43-14.1)$ & $6.5(4.7-14.4)$ & NS \\
\hline Resection only, post-op, $n=55$ & & $8.7(5.5-14.1)$ & $6.25(4.35-11.58)$ & \\
\hline Biopsy only, post-op, $n=10$ & & $8.0(4.5-14.6)$ & $12.85(9.83-18.30)$ & \\
\hline $\begin{array}{l}\text { Absolute Lymphocyte Count on CBC, post-op } \\
\left(x_{10} / \mu L\right), n=67\end{array}$ & $0.96(0.67-1.54)$ & $0.96(0.66-1.59)$ & $0.96(0.68-1.42)$ & NS \\
\hline Resection only, post-op, $n=55$ & & $1.00(0.67-1.61)$ & $0.74(0.65-1.22)$ & \\
\hline Biopsy only, post-op, $n=10$ & & $0.86(0.53-1.66)$ & $1.34(0.93-1.76)$ & \\
\hline Percent Monocyte Count on CBC, post-op, $n=67$ & $6.8(4.8-9.0)$ & $6.1(3.13-8.5)$ & $7.6(5.2-9.4)$ & 0.038 \\
\hline $\begin{array}{l}\text { Absolute Monocyte Count on CBC, post-op } \\
\left(x 10^{3} / \mu \mathrm{L}\right), n=67\end{array}$ & $0.88(0.52-1.11)$ & $0.72(0.44-1.06)$ & $1.01(0.82-1.16)$ & 0.042 \\
\hline Progression-Free Survival (mo) & $5.56(3.02-11.31)$ & $5.03(2.86-8.52)$ & $8.38(3.48-13.28)$ & 0.125 \\
\hline Overall Survival (mo) & $11.23(4.87-20.9)$ & $10.4(4.73-17.67)$ & $17.17(6.67-29.17)$ & 0.085 \\
\hline
\end{tabular}

Abbreviations: IDH: isocitrate dehydrogenase; MGMT: O-6-methylguanine-DNA methyltransferase; EGFR: epidermal growth factor receptor; STR: subtotal resection; NTR: near-total resection; GTR: gross-total resection; CBC: complete blood count; LITT: laser interstitial thermal therapy 
Post op CBC: $n=40$ in no preop steroid group, $n=27$ in yes preop steroid group

NS: $p>0.2$ 